\title{
Women in Technology: Uma análise bibliométrica dos últimos cinco anos
}

\author{
Andréa Sabedra Bordin ${ }^{1}$, Isadora Garcia Ferrão ${ }^{2}$, Kalinka R. L. J. C. Branco ${ }^{2}$ \\ ${ }^{1}$ Universidade Federal de Santa Catarina (UFSC) - Santa Catarina, Brasil. \\ ${ }^{2}$ Universidade de São Paulo (USP) - São Paulo, Brasil \\ andrea.bordin@ufsc.br, isadoraferrao@usp.br, kalinka@icmc.usp.br
}

\begin{abstract}
This paper aims to provide a bibliometric analysis of the five years of the Women in Technology (WIT). The research uses indicators of Productivity and Impact, as well as indicators of Scientific Collaboration. The analysis reveals that the number of papers, authors and citations has increased over the years. On the other hand, the scientif collaborations network appears fragmented, with many groups publishing in isolation. It is possible to conclude that WIT is strengthening itself as a space for sharing experiences and knowledge of an active community interested in the theme of women in technology.
\end{abstract}

Resumo. Este artigo tem como objetivo prover uma análise bibliométrica dos cinco anos do workshop Women in Tehcnology (WIT). A pesquisa utiliza indicadores de Produtividade e Impacto, bem como indicadores de Colaboração Científica. A análise revela que o número de artigos, autores e citações aumentaram no decorrer dos anos. Já a rede de colaboração científica aparece fragmentada, com muitos grupos publicando de forma isolada. É possível concluir que o WIT está se fortalecendo enquanto espaço para compartilhamento de experiências e conhecimento de uma comunidade ativa e interessada na temática mulheres na tecnologia.

\section{Introdução}

O interesse da academia pelo tema mulheres na Computação vem crescendo anualmente. Uma pesquisa pelos termos women e technology nas bases de dados científicas Scopus e ACM Digital Library revelou que o número de artigos e conferências que abordam esse tema vem aumentando. Os eventos científicos têm um papel importante no fomento a essa discussão, pois propiciam um espaço para a troca de experiências e compartilhamento de conhecimento entre pesquisadores, professores, alunos e profissionais da indústria interessados nesta temática. Dessa agregação resultam discussões, trocas e insights que convertem-se em ações diretas e retroalimentam os próprios eventos.

No Brasil, o Women in Technology (WIT) $)^{1}$ é uma iniciativa da Sociedade Brasileira de Computação (SBC) que visa discutir os assuntos relacionados a questões de gênero e a Tecnologia de Informação (TI). O evento concentra-se em debater problemas relacionados à mulher e ao seu acesso à TI, tanto do ponto de vista de mercado de trabalho quanto de inclusão e alfabetização digital.

\footnotetext{
${ }^{1}$ https://csbc.ufsc.br/eventos/wit/
} 
O WIT teve sua gênese em 2007, no Congresso da Sociedade Brasileira de Computação (CSBC), oferecendo por nove anos uma programação focada em palestras convidadas e painéis. Em 2016, juntamente com o programa Meninas Digitais, também da SBC, realizou pela primeira vez a chamada para a submissão de trabalhos apresentados na forma de pôsteres. Com o crescimento do número de projetos nesta área, percebeuse a necessidade de registros dessas ações e experiências [Solis et al. 2016]. Assim, em 2019 foi aberta a chamada para artigos completos com resultados de projetos de pesquisa e extensão, estudos de caso e experiências relevantes ao tema. Os artigos vêm sendo avaliados por um processo de revisão por pares do tipo double blind, evidenciando a busca por trabalhos de qualidade.

Passados cinco anos do início da abertura das chamadas, o WIT já publicou 128 artigos curtos e 46 artigos completos, indicando a existência de uma comunidade coesa e produtiva. Analisar os indicadores bibliométricos de produção científica do evento permite a descoberta de autores mais produtivos, autores com laços de colaboração mais frequentes, instituições de ensino que mais colaboram, dentre outros. O produto final dessa análise possibilita tomadas de decisão, tanto em nível individual (pesquisador), como em nível de gestão da organização do evento. Por exemplo, ao conhecer os autores mais ativos, pesquisadores iniciantes podem estabelecer contato para orientações futuras, assim como a organização do evento pode convidá-los para se envolver na programação e na organização do próprio evento.

Este artigo apresenta uma análise bibliométrica do WIT, a partir de indicadores relacionados à produtividade e colaboração científica dos autores. Foram utilizados indicadores como número de artigos por autor, número de citações, dentre outros. Já em relação à colaboração científica, o método de análise de redes social permitiu obter medidas como grau médio de colaboração, autores que mais colaboram, grupos de autores conectados, entre outras medidas.

O restante deste artigo está organizado da seguinte forma: na Seção 2 são abordados conceitos de análise bibliométrica e um método de análise de colaboração científica; na Seção 3 é descrita a metodologia; os resultados são apresentados e discutidos na Seção 4 e, por fim, as considerações finais são expostas na Seção 5.

\section{Análise bibliométrica}

A bibliometria é uma disciplina que dispõe de técnicas quantitativas e estatísticas de medição dos índices de produção e disseminação do conhecimento científico [Fonseca 1986]. Ela pode ser aplicada no estudo de diversos tipos de produção bibliográfica, como livros, artigos de periódicos e eventos.

Uma vez que essa disciplina pode ser aplicada no estudo de diversos tipos de produções científicas, análises bibliométricas resultam em indicadores bibliométricos. Dentre eles estão os que medem a produtividade científica e tecnológica, como o número de artigos e livros publicados e patentes registradas; os que buscam fazer aproximações quanto ao uso e a qualidade dos documentos, baseados principalmente, nos estudos de citações e os indicadores de colaboração, que analisam redes sociais e colaborativas estabelecidas entre pesquisadores, organizações ou países [Rehn and Kronman 2008]. Cabe salientar que dentre os indicadores, o de análise de colaboração merece destaque, e sendo assim é detalhado na subseção 2.1 . 


\subsection{Análise da colaboração científica}

O indicador mais utilizado nos estudos sobre colaboração científica é a coautoria. Dentre os métodos disponíveis para analisar a colaboração tem-se a Análise de Redes Sociais (ARS) [Newman 2004]. Por meio do método de ARS, os autores de artigos científicos e suas relações de coautoria são modelados em um grafo (rede), onde os nodos (nós, atores) representam os autores e as arestas representam as relações de coautoria.

Através das métricas de ARS é possível identificar em uma rede de coautoria alguns fatos interessantes como, por exemplo, ilhas de colaboração científica, intensidade das relações entre os pesquisadores, autores que mais colaboram com outros autores, dentre outros. Uma rede pode ser analisada em três níveis: de rede, onde propriedades gerais são analisadas; individual, onde as propriedades individuais dos atores são analisadas e de grupo, onde são observadas as formações de subgrupos de atores da rede [Wasserman and Faust 1994].

No nível de análise de rede destaca-se a densidade, que mede o estado de completude de um grafo. Quanto mais nodos estão ligados uns aos outros, mais denso será o grafo. Segundo [Katerndahl 2012], redes com alta densidade indicam uma alta atividade de colaboração. O grau médio de uma rede também é um indicador do nível de colaboração da rede, pois determina o número médio de relacionamentos dos atores.

No nível individual, existem métricas de centralidade que buscam descrever as propriedades de localização de um ator na rede, tais como a centralidade de grau. A centralidade de grau é medida por meio do número de nodos adjacentes a um ator na rede. A centralidade de grau reflete o papel do ator em termos de popularidade e atividade. Em uma rede de coautoria indica o total de autores que publicaram conjuntamente com um determinado autor [Freeman 1978].

Em uma perspectiva de grupo, é possível a identificação de componentes ou subgrafos dentro de uma rede [Wasserman and Faust 1994]. Componentes são subgrafos onde os atores estão conectados por algum caminho possível, mas desconectados dos demais subgrafos. Componente gigante é o nome dado ao subgrafo que contém o maior número de atores conectados. Em uma rede de coautoria a presença de mais de um componente na rede indica a existência de grupos que publicam isoladamente.

\section{Metodologia de pesquisa}

O desenvolvimento deste trabalho passou por três etapas: (1) Coleta de dados, (2) Extração e (3) Análise dos resultados. Essas etapas serão detalhadas nas próximas seções.

\subsection{Coleta}

Nesta etapa os dados que serviram de insumo para as análises foram coletados, sendo eles, os artigos dos anais do WIT do período compreendido entre 2016 a 2020. O período de cinco anos contemplou todas as edições do evento com chamada de trabalhos. A coleta foi realizada manualmente por meio da biblioteca digital da $\mathrm{SBC}^{2}$.

\footnotetext{
${ }^{2}$ https://sol.sbc.org.br/index.php/wit
} 


\subsection{Extração}

Os dados de coautoria, filiação, ano de publicação e título dos artigos foram extraídos manualmente e organizados em tabelas ${ }^{3}$. Os nomes dos autores foram normalizados manualmente, sendo para isso imprescindível conhecer o contexto dos dados, particularmente as relações envolvidas.

Após a normalização, todos os autores foram identificados de forma individual (código e nome), assim como foram processadas todas as relações de coautoria entre os autores, conjuntamente com a frequência da relação (número de vezes que dois autores escreveram conjuntamente). Este processo foi realizado de forma automatizada através da implementação de um algoritmo que gerou a rede de coautoria do evento WIT.

O número de citações dos artigos foi buscado no Google Scholar por meio dos títulos dos artigos, garantindo assim a uniformidade das informações obtidas e que o dados pudessem ser manipulados e analisados.

\subsection{Análise}

As análises bibliométricas realizadas foram organizadas em dois grupos: as que resultaram em indicadores de produtividade e de impacto e as que resultaram em indicadores de colaboração científica. Dentre os indicadores de produtividade e impacto estão o número de artigos por ano, número de artigos por autor e número de citações. Dentre os indicadores de colaboração, têm-se o grau médio de colaboração, autores que mais colaboram e número de componentes (grupos) isolados.

As análises relacionadas a produtividade e impacto foram realizadas manualmente por meio de contagens em planilhas. Já as análises de colaboração foram feitas com o auxílio do software de análise exploratória de dados Gephi ${ }^{4}$.

\section{Resultados}

\subsection{Indicadores de produtividade e impacto}

Ao longo dos últimos cinco anos de WIT, foram publicados 154 artigos ao total, conforme é ilustrado na Figura 1(A). O número de publicações aumentou com o passar do tempo, iniciando com 25 artigos em 2016, seguido de 28 artigos nos anos de 2017 e 2018. Já em 2019 foram publicados 31 e em 2020 totalizaram-se 42 trabalhos publicados.

O aumento mais significativo ocorreu entre os anos de 2019 e 2020. Alguns dos fatores que podem justificar esse aumento são: o evento passar a aceitar não somente trabalhos curtos, mas também completos; o aumento de interesse pelo tema, que tem recebido merecido destaque nos últimos anos e o aumento da visibilidade do evento.

Em 2016 foi publicado o menor número de trabalhos, mas isso não se reflete no número de citações, visto que foi o ano que mais obteve citações, conforme é ilustrado na Figura 1(B). Dentre os primeiros quatro anos analisados, observa-se que 2018 obteve um número significativamente menor, não sendo possível identificar os motivos de tal fato. Já o pequeno número de citações de 2020 deve-se ao fato dos anais do evento terem sido publicados recentemente.

\footnotetext{
${ }^{3}$ http://bit.ly/tabelas-wit-2016a2020

${ }^{4}$ Disponível em: http://gephi.org/
} 


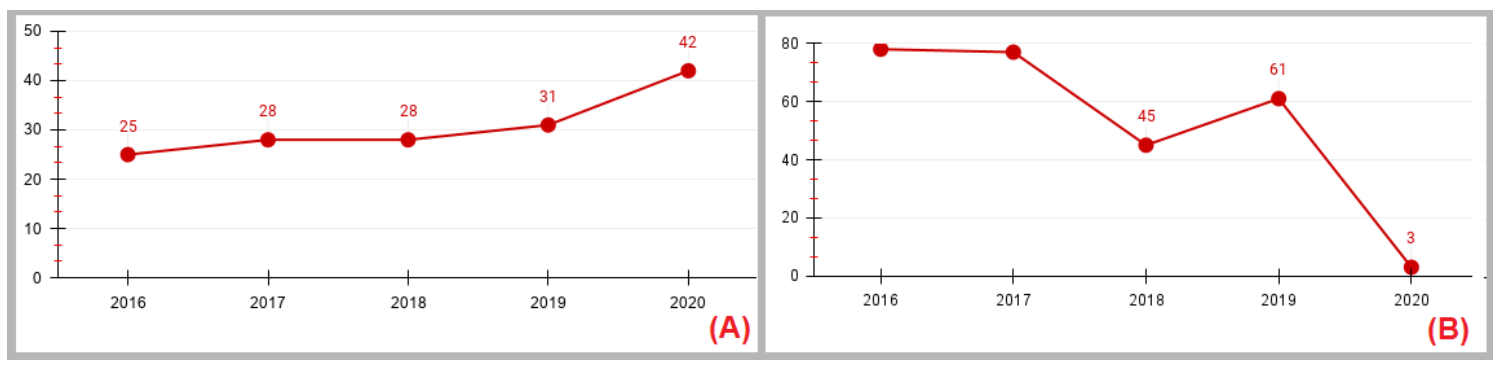

Figura 1. (A) Quantidade total de publicações (B) Quantidade total de citações

Na Tabela 1 é possível observar a quantidade de citações ao longo dos anos categorizadas em: (1) Nenhuma citação; (2) Entre 1 e 3 citações; (3) Entre 4 e 9 citações e (4) 10 ou mais citações. Do total dos trabalhos publicados, 61 (39,3\%) não possuem nenhuma citação, 66 (42,5\%) têm entre 1 a 3 citações, 26 (17\%) possuem de 4 a 9 citações e apenas $2(1,3 \%)$ possuem 10 ou mais citações.

Os dois artigos mais citados foram publicados no WIT de 2016. O trabalho mais referenciado possui 15 citações e é intitulado Cunhantã Digital: programa de incentivo à participação de mulheres da região amazônica na Computação e áreas afins, cujo os autores são filiados à Universidade Federal do Amazonas (UFAM). Este artigo apresenta um programa que desenvolve ações e projetos voltados ao incentivo à participação de mulheres da região amazônica na área de ciência e tecnologia [Lauschner et al. 2016]. Já o segundo trabalho mais referenciado, com 11 citações, é intitulado Ciência da Computação também é coisa de menina, de autoria de filiados à Universidade Federal Fluminense (UFF). Este trabalho descreve a atividade inaugural realizada com os alunos recém matriculados no curso de Ciência da Computação cujo objetivo foi promover uma discussão sobre o que leva as meninas a escolherem ou (não) a área de tecnologia da informação e analisar a interação entre os alunos, principalmente com as meninas, nos primeiros dias de aula [Mochetti et al. 2016].

Tabela 1. Quantidade de citações aos artigos por ano

\begin{tabular}{|c|c|c|c|c|c|c|}
\hline \multirow{2}{*}{ Categoria } & \multicolumn{7}{|c|}{ Quantidade de artigos } \\
\cline { 2 - 7 } & $\mathbf{2 0 1 6}$ & $\mathbf{2 0 1 7}$ & $\mathbf{2 0 1 8}$ & $\mathbf{2 0 1 9}$ & $\mathbf{2 0 2 0}$ & Total \\
\hline Nenhuma citação & 6 & 4 & 5 & 7 & 39 & 61 \\
\hline Entre 1 e 3 & 12 & 13 & 20 & 18 & 3 & 66 \\
\hline Entre 4 e 9 & 5 & 11 & 3 & 6 & 0 & 25 \\
\hline 10 ou mais & 2 & 0 & 0 & 0 & 0 & 2 \\
\hline
\end{tabular}

Não apenas o número de artigos publicados e citações cresceram ao longo dos anos, como também a quantidade de autores interessados em publicar no evento, conforme é apresentado na Tabela 2. Em 2016 haviam 82 autores, já em 2020 esse número quase dobrou, subindo para 155 autores, o que entende-se estar alinhado aos fatores relacionados ao aumento do número de artigos anteriormente apresentados.

Em relação a quantidade de autores por número de publicações, tem-se que 457 autores realizaram apenas uma publicação, com destaque para o ano de 2020 onde 141 novos autores publicaram pela primeira vez. Como pode ser observado na Tabela 2, ao longo de todos os anos do evento, apenas 1 autor realizou 5 ou mais publicações em 
um mesmo ano. A autora, Eliane Pozzebon, da Universidade Federal de Santa Catarina (UFSC) possui, 6 artigos publicados nos anais do WIT, sendo 5 trabalhos em 2016 e 1 trabalho em 2020.

Tabela 2. Quantidade de autores e número de publicações

\begin{tabular}{|c|c|c|c|c|c|c|}
\hline \multirow{2}{*}{ Categoria } & \multicolumn{6}{|c|}{ Quantidade de autores } \\
\cline { 2 - 7 } & $\mathbf{2 0 1 6}$ & $\mathbf{2 0 1 7}$ & $\mathbf{2 0 1 8}$ & $\mathbf{2 0 1 9}$ & $\mathbf{2 0 2 0}$ & Total por quantidade \\
\hline Apenas 1 publicação & 69 & 78 & 88 & 81 & 141 & 457 \\
\hline Entre 2 e 4 publicações & 12 & 12 & 6 & 7 & 14 & 51 \\
\hline 5 ou mais publicações & 1 & 0 & 0 & 0 & 0 & 1 \\
\hline Total por ano & 82 & 90 & 94 & 88 & 155 & 509 \\
\hline
\end{tabular}

Quando se trata dos autores com maior número de publicações ao longo dos cinco anos de evento, observa-se na Figura 2 que Cristiano Maciel e Karen da Silva Figueiredo da Universidade Federal do Mato Grosso (UFMT) lideram o ranking de artigos publicados, totalizando 11 artigos. O número de trabalhos desses dois autores têm uma distribuição muito semelhante, com artigos publicados em todos os anos do evento, com uma diferença apenas entre os anos de 2018 e 2019. Esse expressivo número de publicações é reflexo do envolvimento e trabalho que os autores realizam a frente do programa Meninas Digitais da SBC.

Na segunda posição está Silvia Amélia Bim da Universidade Tecnológica Federal do Paraná (UTFPR) com 9 artigos publicados distribuídos ao longo dos anos, com exceção do ano de 2018. Na sequência aparece Aletéia Patrícia F. de Araujo da Universidade de Brasília (UnB) com 8 artigos, distribuídos em todos os anos. Com 7 artigos publicados estão três autoras: Luciana Bolan Frigo da UFSC, com artigos concentrados na primeira e na última edição; e Maristela de Holanda da UnB e Rosiane de Freitas da Universidade Federal do Amazonas (UFAM), com artigos distribuídos em todos os anos. A seguir vem a autora Eliane Pozzebon da UFSC, com 6 artigos, também concentrados na primeira e na última edição. Fechando o grupo de autores com 5 ou mais artigos publicados estão: Renata V. de Figueiredo e Vanessa Farias Dantas, ambas da Universidade Federal da Paraíba (UFPB), com artigos publicados nos primeiros quatro anos e Aline Vieira de Mello da Universidade Federal do Pampa (Unipampa), com mais artigos publicados nos dois últimos anos.

Ao olhar para as instituições de filiação dos autores que publicaram no WIT, temse um total de 67 instituições, onde $78 \%$ são universidades federais e estaduais, $12 \%$ são universidades privadas, $4 \%$ são escolas de educação básica, 3\% são empresas privadas de tecnologia, 1,5\% corresponde a única empresa federal da área de tecnologia e, por fim, $1,5 \%$ representa uma ONG de mulheres na área de tecnologia.

Na Tabela 3 são apresentadas as 6 instituições com maior quantidade de autores (QAu) e maior quantidade de artigos publicados (QAr), conjuntamente com a proporção média de autores por artigo (QAu/QAr). É possível observar que a UFPB possui o maior número de autores (31), o terceiro maior número de artigos (9) e a maior proporção de autores por artigo $(3,4)$, o que indica um cenário de expressivo interesse pelo tema, assim como de colaboração entre os autores dessa instituição. Por outro lado tem-se a UFMT, com o menor número de autores (15), mas o maior número de artigos (15), resul- 


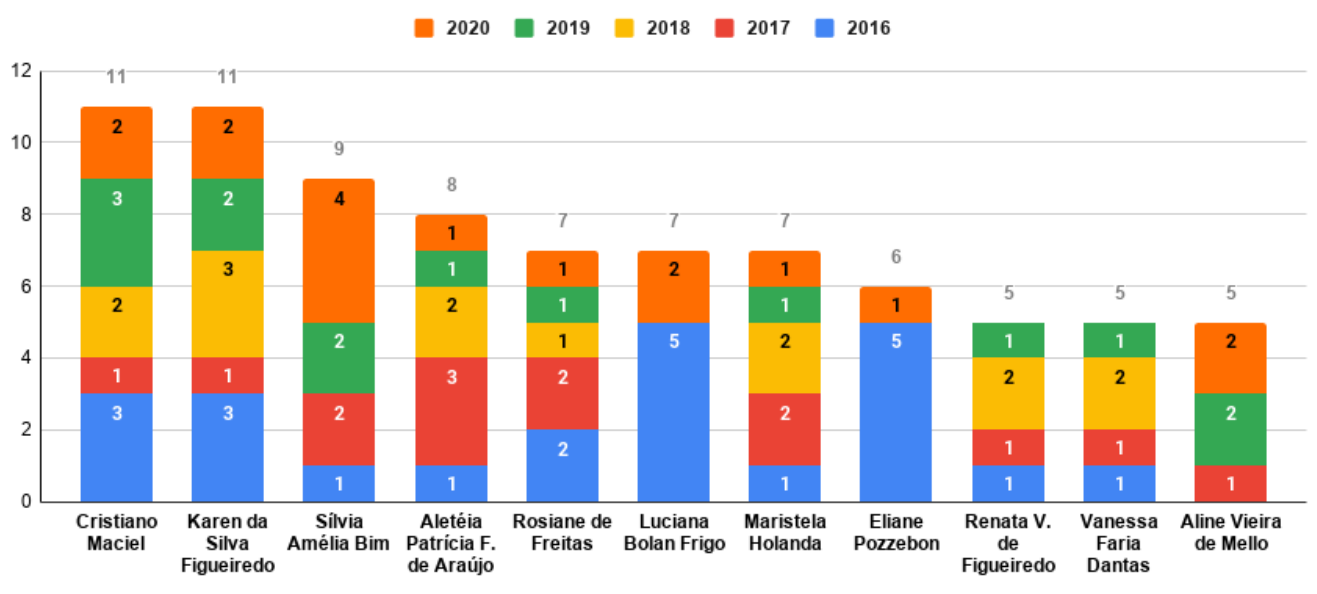

Figura 2. Autores que mais publicaram nos cinco anos do WIT

tando em uma proporção de 1 autor por artigo, indicando mais produtividade, mas menor colaboração entre autores diferentes.

Tabela 3. Universidades que possuem o maior número de autores

\begin{tabular}{|c|c|c|c|}
\hline Universidade & Qtd. Autores (QAu) & Qtd. Artigos (QAr) & QAu/QAr \\
\hline UFPB & 31 & 9 & 3,4 \\
\hline UTFPR & 28 & 15 & 1,8 \\
\hline UFC & 22 & 10 & 2,2 \\
\hline UnB & 17 & 9 & 1,8 \\
\hline UFAM & 16 & 8 & 2,0 \\
\hline UFMT & 15 & 15 & 1,0 \\
\hline
\end{tabular}

\subsection{Indicadores de Colaboração Científica}

A rede de coautoria de artigos das últimas cinco edições do WIT (2016-2020) é composta por 436 nodos (autores) e 999 arestas (relações de coautoria). A Figura 3 fornece uma visão geral da rede, onde cada autor é representado pela cor da instituição de filiação.

Partindo de uma perspectiva de análise da rede como um todo, tem-se que o grau médio é 4,6, o que indica o número médio de colaboradores (coautores) de cada autor na rede. A densidade da rede é 0,01 - onde o valor máximo é 1,0 -, o que indica pouca exploração das possibilidades de colaboração entre os autores. A baixa conectividade pode ser explicada devido a tendência dos artigos serem realizados em parceria com os coordenadores e executores de projetos, que em sua maioria, tem escopo institucional.

Nesta mesma perspectiva, observa-se que a rede possui 70 componentes conectados (subgrafos), evidenciando a existência de grupos de autores conectados por algum caminho, mas desconectados dos demais grupos. Dentre eles, 33 componentes isolados são formados por grupos de 2 a 4 autores; 24 componentes por grupos de 5 a 8 autores; 7 componentes por grupos de 9 a 13 autores; 2 componentes com grupos de 16 e 19 autores e, por fim, 2 componentes com um único autor. O maior componente da rede (componente gigante) é formado por 74 autores. 


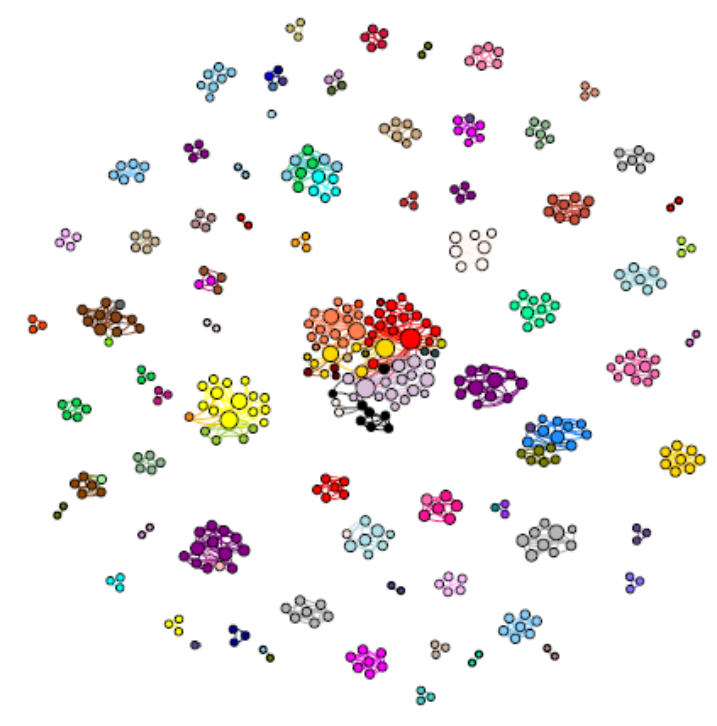

Figura 3. Rede de coautoria do WIT

Como pode ser observado na Figura 3, a grande maioria dos componentes é formado por autores da mesma instituição, denotando uma preferência de colaboração com autores filiados à própria instituição. Em apenas 14 componentes observam-se relações de colaboração interinstitucional, sendo que em 8 deles a relação é entre 2 instituições e nos outros 6 a relação envolve 3 ou mais instituições. É importante destacar que os autores de um componente não necessariamente colaboram (produzem em parceria) entre si, ou seja, eles pertencem ao mesmo grupo porque compartilham algum coautor.

$\mathrm{Na}$ Figura 4 é ilustrado o componente gigante com 74 autores, representando 17\% do total de nodos da rede, onde observam-se que 13 instituições possuem algum laço de colaboração. As instituições com maior representatividade são: UTFPR (18 autores), UFAM (16 autores) e UFSC (13 autores). UFMT e UTFPR são as instituições onde os autores possuem laços de colaboração mais fortes (colaboram com mais frequência). UTFPR é a instituição que colabora com o maior número de autores de outras instituições.

Na perspectiva de análise individual dos nodos (autores) da rede, a Tabela 4 exibe os cinco autores com maior Centralidade de Grau Ponderada (CGP) e Centralidade de Grau (CG) simples, conjuntamente com a instituição a qual são filiados. Observa-se que o mesmo conjunto de autores se destaca em ambas as medidas, que indicam, respectivamente, os autores que mais colaboram com outros autores levando em conta o número de publicações em conjunto (CGP) e o número de relações de coautoria simples (CG).

Tabela 4. Autores com maior centralidade de grau

\begin{tabular}{|c|c|c|c|}
\hline Pesquisador & CGP & CG & Instituição \\
\hline Sílvia Amélia Bim & 34 & 28 & UTFPR \\
\hline Rosiane de Freitas & 30 & 20 & UFAM \\
\hline Cristiano Maciel & 29 & 16 & UFMT \\
\hline Aletéia Patrícia F. de Araújo & 26 & 18 & UnB \\
\hline Luciana Bolan Frigo & 24 & 17 & UFSC \\
\hline
\end{tabular}




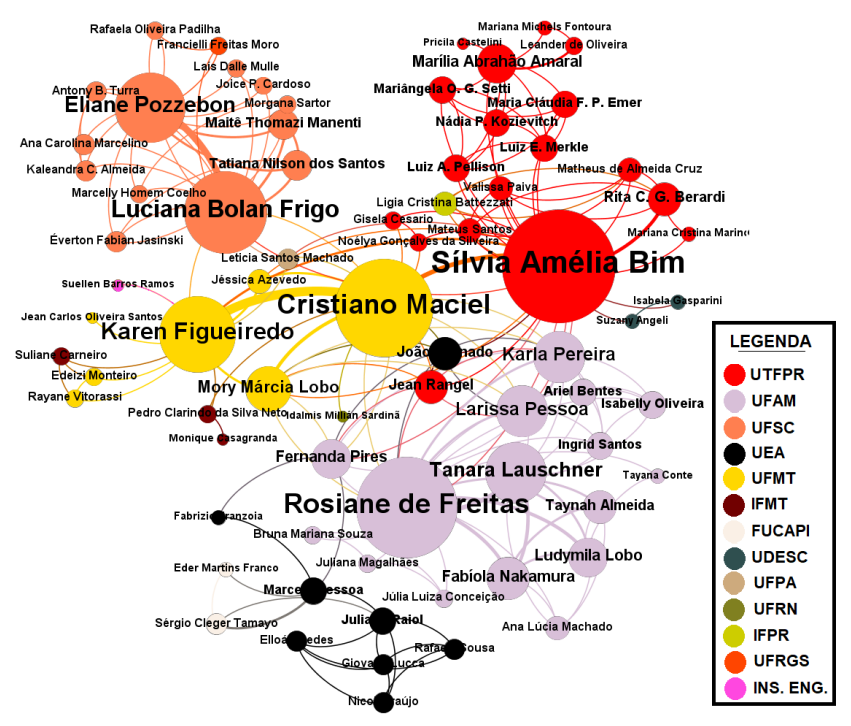

Figura 4. Componente gigante da rede

É possível perceber que alguns autores possuem uma CGP maior que a CG, o que denota que não colaboram com uma quantidade maior de pessoas, mas com mais frequência com as mesmas pessoas. Outro aspecto a ser destacado é que 4 desses autores com forte grau de colaboração pertencem ao componente gigante da rede, como pode ser observado na Figura 4.

Quando se observa a frequência das relações de colaboração, é possível ver na Tabela 5 quais são os autores que mais publicaram conjuntamente. Neste caso, destaca-se que todas as duplas de coautores pertencem a mesma instituição. A frequência mínima é 5 , que é o ponto de corte do número de eventos contemplados neste trabalho.

Tabela 5. Autores com maior frequência de coautoria

\begin{tabular}{|c|c|c|}
\hline Autor A - Instituição & Autor B - Instituição & Frequência \\
\hline Karen da Silva Figueiredo - UFMT & Cristiano Maciel - UFMT & 8 \\
\hline Maristela Holanda - UnB & Aletéia Patrícia F. de Araújo - UnB & 7 \\
\hline Luciana Bolan Frigo - UFSC & Eliane Pozzebon - UFSC & 6 \\
\hline Renata Figueiredo - UFPB & Vanessa Faria Dantas - UFPB & 5 \\
\hline Karina Castelo Branco - UFC & Anna Marques - UFC & 5 \\
\hline
\end{tabular}

\section{Conclusões}

Esse trabalho apresentou uma análise bibliométrica dos artigos publicados nos últimos cinco anos do WIT. Os resultados apresentaram indicadores de produtividade, impacto e colaboração científica, os quais têm potencial para auxiliar a tomada de decisão, tanto em nível estratégico por parte dos comitês de organização do evento, quanto em nível operacional pelos pesquisadores da área.

Os indicadores de produtividade revelaram um volume crescente de artigos e autores, em consonância com os indicadores de impacto, que também mostraram um aumento no número de citações. Entende-se que este cenário positivo tem sua gênese no programa 
Meninas Digitais (MD) da SBC, que incentiva e apoia projetos em todas as regiões do país, resultando em uma comunidade atuante e criativa, que compartilha iniciativas e resultados na forma de publicações, as quais servem como inspiração para a proposição e divulgação de novas iniciativas. Para que este ciclo continue evoluindo, parece ser adequado que as políticas de divulgação e incentivo já estabelecidas pelo MD permaneçam propiciando o mesmo ou um maior nível de engajamento.

Já os indicadores de colaboração científica revelam a existência de uma rede de colaboração fragmentada, onde a maioria dos grupos são compostos por autores da mesma instituição. Neste caso, entende-se que colaboração interinstitucional possa ser mais buscada e incentivada, uma vez que isso propicia que ações e problemas de pesquisa possam ser executados e estudados com olhares e perspectivas diferenciadas, trazendo mais qualidade aos resultados apresentados.

Como ameaça à validade deste trabalho destaca-se a contagem manual de alguns parâmetros, os quais apesar de terem passado por um processo de revisão em várias etapas, podem estar sujeitos a erros. Por outro lado, todo o processo de extração dos dados foi documentado e disponibilizado de forma a propiciar a proveniência dos dados.

Como trabalhos futuros, pretende-se aprofundar as análises bibliométricas, por exemplo, identificando quais tipos de trabalho têm sido apresentados (relatos de projetos de extensão, relatos de experiência de empresas, estudos empíricos, etc.); analisando a rede de citações dos trabalhos publicados no próprio WIT, assim como os veículos que referenciam os artigos do WIT, dentre outros.

\section{Referências}

Fonseca, E. N. d. (1986). Bibliometria: teoria e prática., volume 1. Cultrix, Ed. da USP.

Freeman, L. C. (1978). Centrality in social networks conceptual clarification. Social networks, 1(3):215-239.

Katerndahl, D. (2012). Evolution of the research collaboration network in a productive department. Journal of Evaluation in Clinical Practice, 18(1):195-201.

Lauschner, T., de Freitas, R., Nakamura, F., and Lobo, L. (2016). Cunhantã digital: programa de incentivo a participação de mulheres da região amazônica na computação e áreas afins. In Anais do X Women in Information Technology, pages 20-24. SBC.

Mochetti, K., Salgado, L., Zerbinato, A. V., Souza, B. L., and Avelino, M. R. E. (2016). Ciencia da computação também é coisa de menina! In Anais do X Women in Information Technology, pages 11-15. SBC.

Newman, M. E. (2004). Coauthorship networks and patterns of scientific collaboration. Proceedings of the National Academy of Sciences of the United States of America, 101(1):5200-5205.

Rehn, C. and Kronman, U. (2008). Bibliometric Handbook, volume 1. Karolinska Institutet University Library.

Solis, P. et al. (2016). Abertura do x women in information technology. In $X$ Women in Technology (WIT).

Wasserman, S. and Faust, K. (1994). Social network analysis: Methods and applications, volume 8. Cambridge university press. 